Title:

The Paradox of Stasis and the Nature of Explanations in Evolutionary Biology

\begin{abstract}
(about 120 words):
Recently, a paper by Estes and Arnold claimed to have "solved" the paradox of evolutionary stasis; they claim that stabilizing selection, and only stabilizing selection, can explain the patterns of evolutionary divergence observed over "all timescales". While Estes and Arnold clearly think of their work as identifying the processes that produce evolutionary stasis, close attention to their claims reveal that they do no such thing. Instead, Estes and Arnold identify a particular evolutionary pattern - stabilizing selection as a statistical descriptor - but fail to identify the processes that produce that pattern. This mistake is important; the slippage between pattern and process is common in population and quantitative genetics, and contributes to a persistent misunderstanding of the nature of explanations in evolutionary biology.
\end{abstract}

DRAFT: Please do not cite without author's permission 


\section{The Paradox of Stasis and the Nature of Explanations in Evolutionary Biology ${ }^{1}$}

I. Introduction: Patterns, processes, and the paradox of stasis

In "Resolving the Paradox of Stasis: Models with Stabilizing Selection Explain Evolutionary Divergence on All Timescales” (2007) Estes and Arnold claim to solve a longstanding problem: why, given the substantial amount of heritable phenotypic variation in most populations at most times, do so many populations show so little phenotypic change over evolutionary time periods? More precisely, why, for many of the populations for which sufficient data for evaluation exists, is there only a very slight trend towards increased phenotypic divergence over vastly increasing numbers of generations? Using data from Gingerich (2001), Estes and Arnold note that average phenotypic divergence increases with time only very slowly - by approximately .84 phenotypic standard deviations per million generations in the sample studied! After considering a variety of proposals for addressing this problem that have appeared in the literature, Estes and Arnold argue that, on the basis of fairly straightforward quantitative-genetics models, only stabilizing selection can explain the observed data.

But have they in fact produced an explanation for evolutionary stasis? Estes and Arnold certainly seem to think so - in fact, they claim that "most studies of stasis have focused on the evolutionary pattern without investigating the processes that produce that pattern" and that it is only through confronting quantitative genetic models with data from the real world that one can “explicitly test alternative explanations of stasis” (2007 282). This move is, however, made too

DRAFT: Please do not cite without author's permission 
Jonathan Kaplan $\quad$ Paradox of Stasis \& Nature of Explanation Page 3 quickly. The models Estes and Arnold deploy cannot identify processes at all. Rather, these models serve to provide a formal description of the phenomenon to be explained. As such, Estes and Arnold can reject certain kinds of scenarios, and hence reject certain classes of causal processes. However, the set of processes that are not rejected do not form anything like an explanatory kind - any number of causally quite different processes can lead to the statistical pattern that Estes and Arnold identify as "stabilizing selection.”

The difficulty is that while "stabilizing selection" may sound like a causal process, as the term is used here, it is really nothing of the sort. The debate surrounding possible resolutions to the paradox of stasis therefore provides an excellent entry into the different ways in which the term “selection” gets used (see Matthen and Ariew 2002), the difficulties with treating the models of population genetics as explanatory (see Glymour 2006), and the nature of explanations in evolutionary biology more generally.

II. The paradox of stasis and Estes and Arnold's "resolution”

Estes and Arnold note that there have been a number of proposed solutions to the paradox of stasis, including at least:

1. protracted periods of stabilizing selection (Charlesworth et al. 1982; Lynch 1990),

2. genetic and developmental constraints (Hansen and Houle 2004; Blows and Hoffmann 2005),

3. selective constraints due to coevolution (reviewed in Mayr 2001, chap. 10),

DRAFT: Please do not cite without author's permission 
Jonathan Kaplan $\quad$ Paradox of Stasis \& Nature of Explanation

Page 4

4. canceling of "positive" and "negative" evolutionary trajectories over time (Stanley and Yang 1987; Gingerich 2001),

5. mathematical artifact (Bookstein 1987; Roopnarine 2003),

6. habitat selection (Partridge 1978), and

7. complexities involved with evolution in metapopulations (Eldredge et al. 2005)

(Estes and Arnold 2007 227, numbering added.)

Estes and Arnold produce a variety of quantitative genetics models and compare the outputs of those models to the data-set from Gingerich (2001). Selection, in these models, is about the relationship between the optimum (fittest) phenotype and the current phenotypic makeup of the population. Directional selection is modeled by an optimum that moves in a particular direction, stabilizing selection by an optimum that stays within the current range of phenotypic variation of the population, and various "random walks" modeled by an optimum which moves according to some randomizing algorithm or other. Estes and Arnold argue that, for biologically reasonable values of such variables as the heritability of the phenotypic train in question, the variance of the phenotypic, and the size of the population, the only models that match the empirical data are those in which there is strong stabilizing selection for a phenotypic trait value that remains bounded within a relatively small zone over every time-scale (the “displaced optima” model, 2007 238). From this, Estes and Arnold conclude that the most plausible explanation for stasis is (1) - stabilizing selection.

But have Estes and Arnold shown that only stabilizing selection can in fact account for the patterns observed in nature? Not exactly. Estes and Arnold have shown that the pattern

DRAFT: Please do not cite without author's permission 
Jonathan Kaplan $\quad$ Paradox of Stasis \& Nature of Explanation Page 5 observed in nature matches a particular kind of pattern called "stabilizing selection," a pattern that is defined formally by models with particular "selection" parameters. The difficulty is that while these models are incompatible with certain classes of processes, they are not causal models, and are compatible with too large a range of possible causal processes (see Glymour 2006).

III. Stabilizing selection: Pattern or process?

When Estes and Arnold refer to "stabilizing selection," they are referring to a feature of models. One way of visualizing these models as an adaptive landscape; stabilizing selection becomes a fact about the "shape" of the adaptive landscape (2007 230). The idea is that under those conditions, selection favors a phenotype of intermediate value in the population, and the strength of stabilizing selection is determined by the relationship between the phenotypic variance in the population and the strength of selection. This certainly makes it seem like appealing to stabilizing selection is to make a causal claim - for selection to favor an intermediate phenotype, or for selection to be strong or weak, would seem to involve making a claim about the kinds of processes that a population is subject to.

However, a closer look reveals that this cannot be the case. The parameters used in the models employed by Estes and Arnold are limited to the "target" phenotype of selection, the strength of selection for that target, the population's mean phenotype and associated variance, and the effective population size. So in these models, anything that produces the same

DRAFT: Please do not cite without author's permission 
Jonathan Kaplan $\quad$ Paradox of Stasis \& Nature of Explanation $\quad$ Page 6 population level pattern is modeled in the same way. For example, the model of "stabilizing selection" can account for cases where there is an intermediate phenotype that is "fittest" (stabilizing selection in the traditional sense), where there is heterosis (heterozygotic advantage), or where there is frequency-dependent selection; given the correct assumptions, these cases can all be modeled with the same parameter values. In these models, "selection" is not a particular process in which some actual phenotypes have a propensity to outperform others reproductively; rather, it is a name given to a particular kind of (average/expected) change in the make-up of a population over time.

If stabilizing selection refers to a particular process, then, the models employed cannot be used to test for stabilizing selection, as too many different kinds of processes can produce the same patterns as the models. However, if stabilizing selection is meant to name a particular class of patterns, this ceases to be a problem - the models employed by Estes and Arnold succeed in demonstrating that the pattern observed matches the patterns produced by the model. But if that is the case, then Estes and Arnold have not succeeding in explaining evolutionary stasis, but have instead more precisely characterized the phenomenon to be explained.

Estes and Arnold recognize that a number of different mechanisms are compatible with what they are calling "stabilizing selection" but fail to take seriously the possibility that this might undermine their claim to have explained stasis. In the next section, some of the different processes compatible with Estes and Arnold's results will be explored, with an eye towards distinguishing those processes that might sensibly be called cases of stabilizing selection (as a process) from those that, while they might produce similar patterns, should not be so-identified.

DRAFT: Please do not cite without author's permission 
IV. Selection, populations, constraints...

How do the possible mechanisms behind the observed stability fit into the list of proposed explanations for stasis listed in section II? If all the reasonable mechanisms in fact fit into the "stabilizing selection" subset, then perhaps the set of possible processes identified by the pattern "stabilizing selection" is not so wide as to preclude thinking of that pattern as identifying an explanatory class of processes.

To take a homely example, consider explanations for the high number of traffic accidents on a particular day. If we find that the accidents occurred following a sudden snow storm, we might, reasonably, conclude that what explained the spike in traffic accident was the snowy roadways (especially given what is known about slick roadways, the history of accidents in snow, etc.). Of course, every individual accident that occurred is likely different in key ways, and it may, in the end, be impossible to specify which accidents were "caused" by the snow on the roads, which would have occurred anyway, and what all the other contributing factors into any particular accident might be. But that doesn't make "the snow storm” any less of an explanation for the increased number of traffic accidents. Here, to point out that "the snow storm” does not uniquely identify some set of factors unique to all and only the (excess) accidents that occurred would be churlish. On the other hand, if the day with increased traffic accidents also fell on a holiday on which there had historically been an increased number of accidents due to, say, an increase in number of intoxicated drivers, claiming that this fact might cast some doubt on the "snow" explanation would be reasonable. While untangling the causes 
Jonathan Kaplan

might be difficult in practice, in principle at least the multiple regression analysis would be straightforward, and the settling on an explanation (or on a set of explanations) equally straightforward.

If the class of processes identified by the match between the model Estes and Arnold identify as "stabilizing selection" is more like "a snow storm," it could well be explanatory even if the particulars of the selective regimes faced by the different populations vary (as they must surely do). But if it is more like "a snow storm OR a drinking holiday OR..." then the claim that it is the explanation for stasis is less compelling. One way of making this question more precise is to ask if in fact the models employed by Estes and Arnold can distinguish "stabilizing selection" (\#1 in the above list) from the other 6 possibilities that they note have been advanced in the literature. These will be considered in turn; first, however, it is worth thinking about why the claim that "stabilizing selection" is responsible for long term stasis is, in some ways at least, challenging.

\section{Stabilizing selection}

Estes and Arnold note that long-term stabilizing selection demands that the target of selection not change (much) over very long periods of time; Estes and Arnold interpret this as a situation in which the position of the peak on an adaptive landscape is not changing (or changing only within a small bounded area) most of the time. But this of course presents a problem - why doesn't the peak location change (much) most of the time? More precisely - why are the changes in peak location over very long time periods not much greater than those observed over very short time periods?

DRAFT: Please do not cite without author's permission 
One possibility of course is that the "ordinary" aspects of the external environment (e.g., temperature, etc) ${ }^{2}$ to which the organisms are responding simply are not changing very much. But Estes and Arnold (correctly) reject this, noting that "the universality of environmental change makes persistence in peak position difficult to accept” (2007 240). Again, environmental change (narrowly construed) over long time periods does seem to be greater than the change experienced over short time periods, so the relative stasis experienced by populations would seem hard to reconcile with a focus on the most obvious aspects of the external environment.

But there are a number of other ways in which the environment experienced by organisms might remain stable. For example, it might be that the primary determinants of the selective regimes experienced by organisms are the other organisms surrounding them. If this is the case, then if the biotic communities experienced by organisms remained stable, so too might the selective regime. As Estes and Arnold suggest:

Many lineages experience stable biotic interactions for millions of generations...

It is not unrealistic to assume that lineages situated in such communities experience long-term stability in the position of their adaptive peaks. (2007 240)

If the shape of the adaptive landscape primarily reflects the ecological communities formed, then changes in most "ordinary" environmental variables might not change the shape of the adaptive landscape because they do not sufficiently change the communities.

Of course, the purported stability of biotic communities itself demands explanation. Sterelny, for example, argues that while there is substantial evidence in the palaeo-ecological DRAFT: Please do not cite without author's permission 
Jonathan Kaplan $\quad$ Paradox of Stasis \& Nature of Explanation

Page 10

record for the existence of stable biotic communities, there is little evidence that such communities exist today. He states that:

there seems no doubt that recent ecological associations are not stabilised. They are not systems held close to equilibrium by networks of biological interaction... Flux is the norm. Communities do not have stabilised memberships. They are not insulated from invasion nor bound together in co-adapted networks. Responses to both biological and physical change are individualistic... "on the community level it would seem to be nearly every species for itself" (Valentine and Jablonski 1993: 349).

(Sterelny 2001 457-458). This, Sterelny argues, creates a puzzle - "What explains the fact (if it really is a fact) that a significant array of palaeocommunities were more tightly integrated than Pleistocene and post-Pleistocene communities, and how was that integration maintained..?” (Sterelny 2001 458-459). Without an answer to this question, explanations of stasis in terms of the stability of communities will be less than wholly satisfying. But they would still count as explanations, identifying as they do a narrow class of plausible processes for consideration.

At the very least then, the models that employ "stabilizing selection" in the formal sense related to the form of the adaptive landscape can be made to match a number of different causal scenarios, a number of different ways in which the environment might not vary. If Estes and Arnold could eliminate the other 6 possibilities, they could justifiably claim to have provided some evidence that a process of stabilizing selection - in one form or another - was the best explanation for stasis. But can those explanations listed by Estes and Arnold as alternatives to DRAFT: Please do not cite without author's permission 
stabilizing selection really be excluded?

\section{Genetic and Developmental Constraints}

Estes and Arnold interpret the second explanation listed, "genetic and developmental constraints" as referring to a lack of "appropriate genetic variation" or to "pleiotropic gene action,” and suggest that both can be successfully modeled using very low values for heritability (207 236). But they note that this is an implausible source for stasis given the very high number of generations considered (up to on the order of millions) and the fact that for short time periods there can be substantial change (2007 239).

However, there are other ways of interpreting "genetic and developmental constraints" than simply a lack of heritable variation. For example, the development of integrated, coherent phenotypes may place limits on the kinds of changes that any particular trait can undergo in a single kind of population. Estes and Arnold note that the "long-term persistence in the position and configuration of the adaptive landscape may be promoted by interactions among characters" and that these kinds of "complex phenotypic interactions help to build the genetic and phenotypic correlations that create phenotypic integration” (2007 240). Indeed, Estes and Arnold argue that:

Stabilizing selection arises from the interaction between organisms and their environment—both internal and external. Stabilizing selection can thus be thought of as resulting from external, normalizing forces (e.g., predation, competition) together with the internal, stabilizing constraints that abound at all levels of DRAFT: Please do not cite without author's permission 
Jonathan Kaplan $\quad$ Paradox of Stasis \& Nature of Explanation

Page 12

organization as a consequence of functional interactions. (2007 240)

But if "stabilizing selection" can arise from "internal stabilizing constraints" then in what sense can "stabilizing selection” and “developmental constraints” possibly count as different explanations for stasis, as Estes and Arnold suggest (2007 227)?

Consider the simple case of heterozygotic advantage provided by malaria resistance and the HbS allele. Famously, in areas with endemic malaria, the HbS allele is kept at a reasonably high frequency by the balance between its role in providing malaria resistance in heterozygotes and its relationship to sickle-cell disease in homozygotes. But is this really best thought of as a case of "stabilizing selection"? Selection consistently favors the resistant phenotype, and there is consistent selection against the sickle-cell disease; there is no intermediate phenotype that is the target of selection. Selection cannot be wholly effective in this case because the trait in question is not heritable in the kind of straightforward way that would permit the trait to go to fixation in the population. But that doesn't mean that selection is in fact selecting for some other trait.

Modeled as an adaptive landscape, where points represent populations with particular allelic frequencies, it is easy to see that the case of heterosis will be just like the case in which selection actually favors an intermediate phenotype. In both cases, a particular allelic frequency in the population will have the highest average fitness.

Note too that if one is tempted to argue that in fact in the $\mathrm{Hb} / \mathrm{HbS}$ case selection does favor an intermediate phenotype - one with some malformed and some normal hemoglobin consideration of frequency dependent selection on phenotypes can be used to yield the same result. Consider the "Hawk-Dove” game, a traditional example of frequency dependent

DRAFT: Please do not cite without author's permission 
Jonathan Kaplan $\quad$ Paradox of Stasis \& Nature of Explanation

Page 13

selection $^{3}$. If we imagine that the Hawk-Dove trait is controlled by a single gene with two alleles (h and d, say), such that the hh and hd genotypes are "Hawks" and dd genotypes "Doves," then selection will maintains an equilibrium of mixed alleles by selecting for a particular frequency of phenotypes. The adaptive landscape will of course be exactly the same (given the correct parameters), and this will still count as “stabilizing selection” for Estes and Arnold, despite the fact that in this case there simply is no intermediate phenotype available for selection to favor.

The models employed by Estes and Arnold, then, cannot distinguish between (some) explanations in terms of "developmental constraints" and "stabilizing selection" - what Estes and Arnold call stabilizing selection encompasses at least some cases of developmental constraints, as well as radically different kinds of processes that might lead to stable allelic frequencies in a population.

\section{Selective Constraints Due to Co-Evolution}

Organisms that are co-evolving might be prevented from undergoing some kinds of evolutionary change because of the demands of the other (kind of) organism. For example, Mayr (2001) discusses the interaction between myxomatosis virus and the rabbit population in Australia; after some time, the virus had settled into a less-lethal form, and the rabbits had developed moderate resistance (211). But note that both virus and rabbit are limited in the evolutionary changes open to them - a change that left the rabbits more susceptible, or the virus far more (or less) virulent, would be selected against whatever its other features.

In some ways, this would seem to be simply a more specific case of the stable ecological DRAFT: Please do not cite without author's permission 
communities explanation discussed above; if that is so, then, by Estes and Arnold's arguments, it shouldn't count as a separate explanation opposed to “stabilizing selection” but is rather just another example of "stabilizing selection." In any event, the only way to model this given the parameters available is as selection for a particular intermediate phenotypic value that does not change (much). The models employed cannot distinguish this from stabilizing selection (understood as a process that is really about the selection of intermediate phenotypes).

4. The canceling of "positive" and "negative" evolutionary trajectories over time Here the models deployed by Estes and Arnold do in fact permit (some versions of) this hypothesis to be tested. They found that there were no reasonable values that can be assigned to the key parameters in their model that permit the models to reproduce the historical data under the assumption that selection favors an optimum phenotype that changes (more or less randomly) over time. There may be room to quibble with the models and the assumptions built into them, but Estes and Arnold present strong and compelling evidence against various kinds of "random walk" models ${ }^{4}$.

Part of what this reveals is that there is nothing in principle wrong with taking seriously the implications of the models that Estes and Arnold present. These models can in fact be used to rule out possible explanations. If they were able to rule out a larger variety of explanations, leaving only one or a very few reasonable explanations left, the claim that they had resolved the problem of evolutionary stasis would be reasonable. The problem seems to emerge, again, from a confusion about just what stabilizing selection, as they understand it, actually is.

DRAFT: Please do not cite without author's permission 
5. Mathematical artifact

Estes and Arnold are at pains to show that no version of a random walk (see 4, above) can explain the taxonomic data. This is important, because unless the hypothesis that the trait in question is evolving as a random walk can be rejected, it is difficult to accurately calculate the rate of evolution in that lineage, and the possibility of linking short and long time-scale changes is much reduced (see Roopnarine 2003). By rejecting random walks, Estes and Arnold avoid the problems pointed towards here (but perhaps Estes and Arnold are too quick to reject random walks in at least some cases - see note 3).

\section{Habitat selection}

Another approach to the problem of evolutionary stasis emerges from an argument that the phenomenon is rather less general than the fossil record suggests. In "The dynamics of evolutionary stasis”(2005) Eldredge et al note that for a novel phenotype to be preserved in the fossil record, it "must originate, become established in a local population, and then spread and increase in numbers across a large geographic area” (136). So the failure to observe a novel phenotype (the failure to observe deviations from stasis) may not be because the phenotype never arose, but simply because it never became established in a large, wide-ranging population. Eldredge et al suggest that this may be due to population structure (this possibility will be dealt with in more detail below, at 7), but more simply, a population that is wide-ranging (for at least some of its evolutionary history) may be insulated from environmental change by the ability to "select" locations with favorable environmental conditions. A population may not change not 
because the environment (in some grand sense) isn't changing, but because the population is actively tracking a particular kind of environment.

This explanation - a kind of migration - would of course be modeled by Estes and Arnold as an example of an adaptive landscape that isn't changing, and hence as an example of stabilizing selection. But it is worth noting that insofar as we wish to call this an example of stabilizing selection at all, it is clearly stabilizing selection of a different sort than the simple case of a wholly exogenous environmental stability. And again, Estes and Arnold list habitat selection as one of a number of alternative possible explanations of stasis. If habitat selection is really just a form of stabilizing selection, then it is hardly an alternative explanation. If, on the other hand, stabilizing selection is supposed to be an alternative to habitat selection, then stabilizing selection cannot refer just to the shape of the adaptive landscape, but must refer to a rather more limited set of causal processes.

7. The complexities involved with evolution in metapopulations

As noted above, Eldredge et al. (2005) argue that the phenomenon of evolutionary stasis can be explained by the structure of populations. Essentially, Eldredge et al suggest that the only species represented in the fossil record are those that had a relatively large range, and hence were likely composed of (many) smaller populations. In the smaller populations, directional selection could act on particular traits, but, when the smaller populations rejoined the larger metapopulations (or went extinct), those changes would be lost.

The models employed by Estes and Arnold do not take specific account of population structure, nor is it at all clear how they could. In any event, from the standpoint of those models, DRAFT: Please do not cite without author's permission 
Jonathan Kaplan $\quad$ Paradox of Stasis \& Nature of Explanation

Page 17

the situation envisioned by Eldredge et al is very much like that envisioned in the habitat selection explanation (6), and as such, would be modeled (again) as a case of stabilizing selection.

In short, the models employed by Estes and Arnold are able to rule out some alternative explanations for stasis, but are faced with a dilemma with respect to other possible "alternatives." If the other explanations are really alternatives to stabilizing selection, the models cannot distinguish between them, and what the model calls "stabilizing selection" is really no such thing. If the other explanations are really just more instances of stabilizing selection, then stabilizing selection no longer refers to selection for an intermediate phenotype, and no longer names a class of possible explanatory processes.

\section{Conclusions: The Heart of Evolutionary Biology?}

In “Wayward Modeling: Population Genetics and Natural Selection” (2006) Glymour argues that most biologists and philosophers have accepted population genetics as providing "the core formal machinery for describing and understanding natural selection and the evolutionary events it produces" (369). On this view, the equations at the heart of population genetics are at the heart of evolutionary biology - they tell us what selection is and when it is occurring.

If this view were correct, we would be forced to accept that the models employed by Estes and Arnold did successfully identify "stabilizing selection” as occurring, and hence did in fact provide an explanation for evolutionary stasis. The fact that they think they have (and that

DRAFT: Please do not cite without author's permission 
Jonathan Kaplan $\quad$ Paradox of Stasis \& Nature of Explanation

Page 18

many biologists seem to agree - see e.g. Hendry 2007, Carroll et al 2007) reveals their commitment to this way of thinking about evolutionary biology.

But if the above is correct, there are good reasons to suggest that Estes and Arnold have not in fact produced an explanation for stasis. If Glymour is correct in arguing that "population genetics models evolving populations with the wrong variables related by the wrong equations employing the wrong kinds of parameters” (2006 371) this should hardly come as a surprise. The problem is that there is a critical and often overlooked distinction between the formal models provided by populations genetics and the causal relationships between particular kinds of physical processes, particular traits, and the reproduction and development of organisms within populations (see also Pigliucci and Kaplan 2006 esp. chapters 1 and 2). The former deal with the statistical patterns produced, whereas the latter deal with the causes generated by organisms in their ecological contexts. Explaining a particular phenomenon involves, at least, appealing to the kind of causal processes that produce that phenomenon, and that is something that appeals to the models employed by population and quantitative genetics simply cannot do.

The solution to the problem of evolutionary stasis still awaits.

DRAFT: Please do not cite without author's permission 
Jonathan Kaplan $\quad$ Paradox of Stasis \& Nature of Explanation

Page 19

References:

Carroll, S.P., A. P. Hendry, D. N. Reznick, and C. W. Fox. 2007. "Evolution on ecological timescales” Functional Ecology 21: 387-393

Eldredge, N., J. N. Thompson, P. M. Brakefield, S. Gavrilets, D. Jablonski, J. B. C. Jackson, R. E. Lenski, B. S. Lieberman, M. A. McPeek, and W. Miller III. 2005. “The dynamics of evolutionary stasis.” Paleobiology 31:133-145.

Estes, S. and S.J. Arnold. 2007. "Resolving the Paradox of Stasis: Models with Stabilizing Selection Explain Evolutionary Divergence” The American Naturalist 169(2): 227-244.

Gingerich, P. D. 2001. "Rates of evolution on the time scale of the evolutionary process." Genetica 112/113:127-144.

Glymour, B. 2006. "Wayward Modeling: Population Genetics and Natural Selection.” Philosophy of Science 73:369-389

Hendry, A. 2007. “The Elvis paradox.” Nature 446(8):147-150

DRAFT: Please do not cite without author's permission 
Jonathan Kaplan $\quad$ Paradox of Stasis \& Nature of Explanation $\quad$ Page 20

Hunt, G. 2007. “The relative importance of directional change, random walks, and stasis in the evolution of fossil lineages.” Proceedings of the National Academy of the Sciences 104(47):

18404-18408

Lewontin, R. 1983/1985. “The Organism as the Subject and Object of Evolution.” Sciencia 118:

63-82. Reprinted in The Dialectical Biologist (Richard Levins and Richard Lewontin) 1985 Harvard University Press, Cambridge, MA.

Matthen, M. and A. Ariew. 2002. “Two Ways of Thinking about Fitness and Natural Selection.” The Journal of Philosophy 99:55-83.

Mayr, E. 2001. What evolution is. Weidenfeld \& Nicholson, London.

Pigliucci, M. and J. Kaplan. 2006. Making Sense of Evolution. Chicago University Press, Chicago.

Roopnarine, P. D. 2003. “Analysis of rates of morphologic evolution.” Annual Review of Ecology and Systematics 34:605-632

Sterelny, K. 2001. “The Reality of Ecological Assemblages: A Palaeo-ecological Puzzle.” Biology and Philosophy 16: 437-461.

DRAFT: Please do not cite without author's permission 
NOTES:

${ }^{1}$ Acknowledgments suppressed for review.

${ }^{2}$ As for example Lewontin (1983/1985) notes, there are deep problems with the idea of an “external environment” to which populations of organisms are simply exposed. However, these problems can be bracketed for now.

${ }^{3}$ In competition for resources, “doves” make a trivial display but back down if threatened, and "hawks" fight for the resource. When two doves interact, each has a 50\% chance of getting the resource without risk, when two hawks interact, each has a 50\% chance of getting the resource, but a high chance of suffering a costly injury, and when a hawk and a dove interact, the hawk always gets the resource. By assigning particular values to the resources, it is easy to get stable equilibrium populations with any hawks/doves frequency one pleases.

${ }^{4}$ But see Hunt 2007, who, employing much the same models but using a different data-set, argues that, with respect to size at least, there is substantial evidence for random walks in the fossil record (Hunt argues that they are roughly as common as stasis).

DRAFT: Please do not cite without author's permission 